\title{
Tractable Latent State Filtering for Non-Linear DSGE Models Using a Second-Order Approximation and Pruning
}

\author{
Robert Kollmann
}

Accepted: 25 December 2013 / Published online: 1 February 2014

(C) Springer Science+Business Media New York 2014

\begin{abstract}
This paper develops a novel approach for estimating latent state variables of Dynamic Stochastic General Equilibrium (DSGE) models that are solved using a second-order accurate approximation. I apply the Kalman filter to a state-space representation of the second-order solution based on the 'pruning' scheme of Kim et al. (J Econ Dyn Control 32:3397-3414, 2008). By contrast to particle filters, no stochastic simulations are needed for the deterministic filter here; the present method is thus much faster; in terms of estimation accuracy for latent states it is competitive with the standard particle filter. Use of the pruning scheme distinguishes the filter here from the deterministic Quadratic Kalman filter presented by Ivashchenko (Comput Econ, 43:71-82, 2014). The filter here performs well even in models with big shocks and high curvature.
\end{abstract}

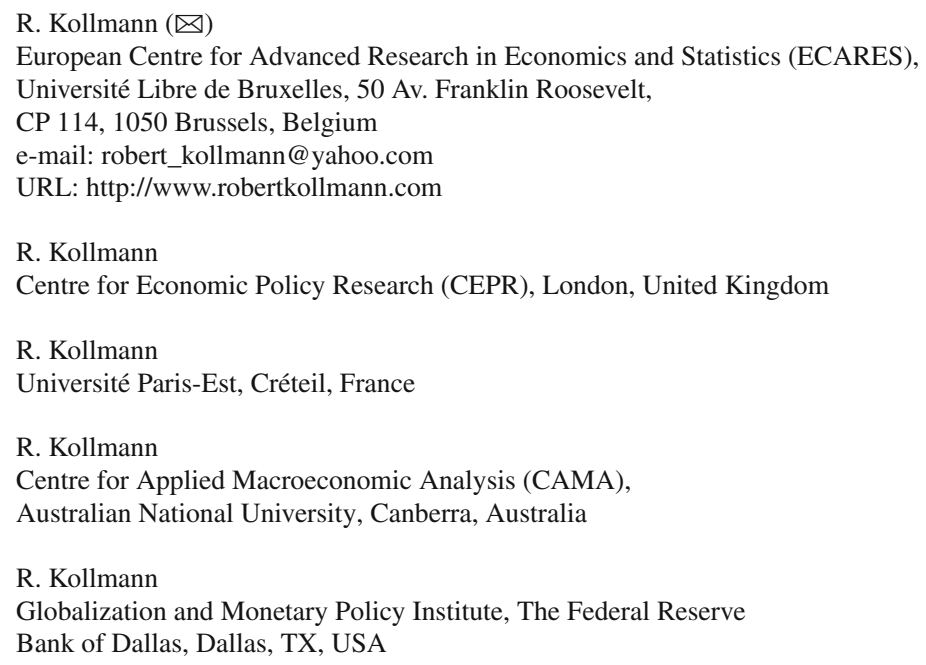


Keywords Latent state filtering - DSGE model estimation .

Second-order approximation · Pruning · Quadratic Kalman filter

JEL Classification $\quad$ C63 $\cdot$ C68 $\cdot$ E37

\section{Introduction}

Dynamic Stochastic General Equilibrium (DSGE) models typically feature state variables that cannot directly be measured empirically (such as preference shocks), or for which data include measurement error. A vast literature during the past two decades has taken linearized DSGE models to the data, using likelihood-based methods (e.g., Smets and Wouters 2007; Negro and Schorfheide 2011). Linearity (in state variables) greatly facilitates model estimation, as it allows to use the standard Kalman filter to infer latent variables and to compute sample likelihood functions based on prediction error decompositions. However, linear approximations are inadequate for models with big shocks, and they cannot capture the effect of risk on economic decisions and welfare. Non-linear approximations are thus, for example, needed for studying asset pricing models and for welfare calculations in stochastic models. Recent applied macroeconomic research has begun to take non-linear DSGE models to the data. This work has mainly used particle filters, i.e. filters that infer latent states using Monte Carlo methods. ${ }^{1}$ Particle filters are slow computationally, which limits their use to small models.

This paper develops a novel deterministic filter for estimating latent state variables of DSGE models that are solved using a second-order accurate approximation (as derived by Jin and Judd 2000, Sims 2000, Collard and Juillard 2001, Schmitt-Grohé and Uribe 2004, Kollmann 2004 and Lombardo and Sutherland 2007). That approximation provides the most tractable non-linear solution technique for medium-scale models, and has thus widely been used in macroeconomics (see Kollmann 2002 and Kollmann et al. 2011 for detailed references). When simulating second-order approximated models, it is common to use the 'pruning' scheme of Kim et al. (2008), under which second-order terms are replaced by products of the linearized solution. Unless the pruning algorithm is used, second-order approximated models often generate exploding simulated time paths. Pruning is therefore crucial for applied work based on second-order approximated models. This paper hence assumes that the pruned secondorder approximated model is the true data generating process (DGP). The method presented here exploits the fact that the pruned system is linear in a state vector that consists of variables solved to second- and first-order accuracy, and of products of firstorder accurate variables. The pruned system thus allows convenient closed-form determination of the one-period-ahead conditional mean and variance of the state vector. I apply the linear updating rule of the standard Kalman filter to the pruned state equation.

The filter here is much faster than particle filters, as it is not based on stochastic simulations. In Monte Carlo experiments, the present filter generates more accurate estimates of latent state variables than the standard particle filter, especially with big shocks or when the model has high curvature. The filter here is also more accurate than

\footnotetext{
${ }^{1}$ See Fernández-Villaverde and Rubio-Ramírez (2007) and An and Schorfheide (2007) for early applications.
} 
a conventional Kalman filter that treats the linearized model as the true DGP. ${ }^{2}$ Due to its high speed, the filter presented here is suited for the estimation of structural model parameters; a quasi-maximum likelihood procedure can be used for that purpose.

This paper is complementary to Andreasen (2012) and Ivashchenko (2014) who also develop deterministic filters for second-order approximated DSGE models, and show that those filters can outperform particle filters. These authors too assume linear updating rules. The filter here is closest to Ivashchenko's (2014) 'Quadratic Kalman filter' $(\mathrm{QKF})$ that is also based on closed-form one-step-ahead conditional moments of the state vector-the key difference is that the QKF does not use the pruning scheme. ${ }^{3}$ The present filter (based on pruning) performs well even in models with big shocks and high curvature-for such models the QKF may generate filtered state estimates that diverge explosively from true state variables. Such stability issues never arose for the filter proposed here, in a wide range of numerical experiments. In models with small shocks and weak curvature the filter developed here and the QKF have similar performance. The present paper is also related to Andreasen et al. (2013) who likewise derive a pruned state-space representation for second-order approximated DSGE models and show how to compute moments for pruned models; these authors develop a method of moments estimator for DSGE models, but do not present a filter for latent state variables. (I learnt about Ivashchenko 2014 and Andreasen et al. 2013 after the present research had been completed.)

\section{Model Format and Filter}

\subsection{Model Format and Second-Order Solution}

Many widely-used DSGE models can be expressed as:

$$
E_{t} G\left(\Omega_{t+1}, \Omega_{t}, \varepsilon_{t+1}\right)=0
$$

where $E_{t}$ is the mathematical expectation conditional on date $t$ information; $G$ : $R^{2 n+m} \rightarrow R^{n}$ is a function, and $\Omega_{t}$ is an $n \times 1$ vector of endogenous and exogenous variables known at $t ; \varepsilon_{t+1}$ is an $m \times 1$ vector of serially independent innovations to exogenous variables. In what follows, $\varepsilon_{t}$ is Gaussian: $\varepsilon_{t} \sim N\left(0, \xi^{2} \Sigma_{\varepsilon}\right)$, where $\xi$ is a scalar that indexes the size of shocks. The solution of model (1) is a "policy function" $\Omega_{t+1}=F\left(\Omega_{t}, \varepsilon_{t+1}, \xi\right)$, such that $E_{t} G\left(F\left(\Omega_{t}, \varepsilon_{t+1}, \xi\right), \Omega_{t}, \varepsilon_{t+1}\right)=0 \forall \Omega_{t}$. This paper focuses on second-order accurate model solutions, namely on second-order Taylor series expansions of the policy function around a deterministic stead state, i.e.

\footnotetext{
2 The literature has discussed 'Extended Kalman filters', i.e. Kalman filters applied to linear approximations of non-linear models; e.g., Harvey (1989).

3 One-step-ahead moments in the QKF are derived under the assumption that estimation error of filtered states is Gaussian. The filter here does not require that assumption. Ivashchenko (2014) also applies two other deterministic filters to second-order approximated DSGE models: a Central Difference Kalman filter (Norgaard et al. 2000) and an Unscented Kalman filter (Julier and Uhlmann 2004); these filters are based on different deterministic numerical integration schemes for computing one-step-ahead conditional moments (no analytical closed-form expressions). Andreasen (2012) estimates a DSGE model using a Central Difference Kalman filter.
} 
around $\xi=0$ and a point $\Omega$ such that $\Omega=F(\Omega, 0,0)$. Let $\omega_{t} \equiv \Omega_{t}-\Omega$. For a $q \times 1$ column vector $x$ whose $i$-th element is denoted $x^{i}$, let

$P(x) \equiv \operatorname{vech}\left(x x^{\prime}\right)=\left(\left(x^{1}\right)^{2}, x^{1} x^{2}, \ldots, x^{1} x^{q},\left(x^{2}\right)^{2}, x^{2} x^{3}, \ldots, x^{2} x^{q}, \ldots,\left(x^{q-1}\right)^{2}, x^{q-1} x^{q},\left(x^{q}\right)^{2}\right)$,

be a column vector consisting of all squares and cross-products of the elements of $x .^{4}$ The second-order accurate model solution can be written as

$$
\omega_{t+1}=F_{0} \xi^{2}+F_{1} \omega_{t}+F_{2} \varepsilon_{t+1}+F_{11} P\left(\omega_{t}\right)+F_{12} \cdot\left(\omega_{t} \otimes \varepsilon_{t+1}\right)+F_{22} P\left(\varepsilon_{t+1}\right),
$$

where $F_{0}, F_{1}, F_{2}, F_{11}, F_{12}, F_{22}$ are vectors/matrices that are functions of structural model parameters, but that do not depend on $\xi$ (Sims 2000; Schmitt-Grohé and Uribe 2004). The first-order accurate (linearized) model solution is:

$$
\omega_{t+1}^{(1)}=F_{1} \omega_{t}^{(1)}+F_{2} \varepsilon_{t+1}
$$

The superscript ${ }^{(1)}$ denotes a variable solved to first-order accuracy. It is assumed that all eigenvalues of $F_{1}$ are strictly inside the unit circle, i.e. that the linearized model is stationary.

\subsection{Pruning}

As discussed above, I use the 'pruning' scheme of Kim et al. (2008) under which second-order terms are replaced by products of the linearized solution-i.e. $P\left(\omega_{t}\right)$ and $\omega_{t} \otimes \varepsilon_{t+1}$ are substituted by $P\left(\omega_{t}^{(1)}\right)$ and $\omega_{t}^{(1)} \otimes \varepsilon_{t+1}$, respectively. With pruning, the solution (2) is thus replaced by:

$$
\omega_{t+1}=F_{0} \xi^{2}+v F_{1} \omega_{t}+F_{2} \varepsilon_{t+1}+F_{11} P\left(\omega_{t}^{(1)}\right)+F_{12} \omega_{t}^{(1)} \otimes \varepsilon_{t+1}+F_{22} P\left(\varepsilon_{t+1}\right) .
$$

Note that $P\left(\omega_{t}\right)=P\left(\omega_{t}^{(1)}\right)$ and $\omega_{t} \otimes \varepsilon_{t+1}=\omega_{t}^{(1)} \otimes \varepsilon_{t+1}$ hold, up to second-order accuracy. ${ }^{5}$ Thus, (4) is a valid second-order accurate solution. In repeated applications of (2), third and higher-order terms of state variables appear; e.g., when $\omega_{t+1}$ is quadratic in $\omega_{t}$, then $\omega_{t+2}$ is quartic in $\omega_{t}$; pruning removes these higher-order terms. The motivation for pruning is that (2) has extraneous steady states (not present in the original model)-some of these steady states mark transitions to unstable behavior. Large shocks can thus move the model into an unstable region. Pruning overcomes this problem. If the first-order solution is stable, then the pruned second-order solution

\footnotetext{
${ }^{4}$ For a square matrix $M$, vech $(M)$ is the column vector obtained by vertically stacking the elements of $M$ that are on or below the main diagonal.

$5 \omega_{t}=\omega_{t}^{(1)}+R^{(2)}$ where $R^{(n)}$ contains terms of order $n$ or higher in deviations from steady state. Let $\omega_{t}^{i}$ and $\omega_{t}^{(1), i}$ be the $i$-th elements of $\omega_{t}$ and $\omega_{t}^{(1)}$, respectively. Note that $\omega_{t}^{i} \omega_{t}^{j}=\left(\omega_{t}^{(1), i}+R^{(2)}\right)\left(\omega_{t}^{(1), j}+R^{(2)}\right)=$ $\omega_{t}^{(1), i} \omega_{t}^{(1), j}+\omega_{t}^{(1), i} R^{(2)}+\omega_{t}^{(1), j} R^{(2)}+R^{(4)}$; thus, $\omega_{t}^{i} \omega_{t}^{j}=\omega_{t}^{(1), i} \omega_{t}^{(1), j}+R^{(3)}$. Up to $2^{\text {nd }}$ order accuracy, $\omega_{t}^{i} \omega_{t}^{j}=\omega_{t}^{(1), i} \omega_{t}^{(1), j}$ and $P\left(\omega_{t}\right)=P\left(\omega_{t}^{(1)}\right)$ holds thus. By the same logic, $\omega_{t} \otimes \varepsilon_{t+1}=\omega_{t}^{(1)} \otimes \varepsilon_{t+1}$ holds to $2^{\text {nd }}$ order accuracy. See Kollmann (2004) and Lombardo and Sutherland (2007).
} 
(4) too is stable. The subsequent discussion assumes that the true DGP is given by the pruned system (3),(4).

\subsection{Augmented State Equation}

The law of motion of $P\left(\omega_{t}^{(1)}\right)$ can be expressed as $P\left(\omega_{t+1}^{(1)}\right)=K_{11} P\left(\omega_{t}^{(1)}\right)+K_{12} \omega_{t}^{(1)} \otimes \varepsilon_{t+1}+K_{22} P\left(\varepsilon_{t+1}\right)$, where $K_{11}, K_{12}, K_{22}$ are matrices that are functions of $F_{1}$ and $F_{2}$. Stacking this matrix equation, as well as (3) and (4) gives the following state equation:

$$
\begin{aligned}
{\left[\begin{array}{l}
\omega_{t+1}\left(\omega_{t+1}^{(1)}\right) \\
\omega_{t+1}^{(1)}
\end{array}\right]=} & {\left[\begin{array}{l}
F_{0} \xi^{2} \\
0 \\
0
\end{array}\right]+\left[\begin{array}{lll}
F_{1} & F_{11} & 0 \\
0 & K_{11} & 0 \\
0 & 0 & F_{1}
\end{array}\right]\left[\begin{array}{l}
\omega_{t} \\
P\left(\omega_{t}^{(1)}\right) \\
\omega_{t}^{(1)}
\end{array}\right]+\left[\begin{array}{l}
F_{2} \\
0 \\
F_{2}
\end{array}\right] \varepsilon_{t+1} } \\
& +\left[\begin{array}{l}
F_{12} \\
K_{12} \\
0
\end{array}\right]\left(\omega_{t}^{(1)} \otimes \varepsilon_{t+1}\right)+\left[\begin{array}{l}
F_{22} \\
K_{22} \\
0
\end{array}\right] P\left(\varepsilon_{t+1}\right) .
\end{aligned}
$$

(5) can be written as: $Z_{t+1}=g_{0}+G_{1} Z_{t}+G_{2} \varepsilon_{t+1}+G_{12}\left(\omega_{t}^{(1)} \otimes \varepsilon_{t+1}\right)+G_{22} P\left(\varepsilon_{t+1}\right)$, with $Z_{t+1} \equiv\left(\omega_{t+1}^{\prime}, P\left(\omega_{t+1}^{(1)}\right)^{\prime}, \omega_{t+1}^{(1) \prime}\right)^{\prime}$, while $g_{0}, G_{1}, G_{2}, G_{12}$ and $G_{22}$ are the first to fifth coefficient vectors/matrices on the right-hand side of (5), respectively. Thus,

$$
Z_{t+1}=G_{0}+G_{1} Z_{t}+u_{t+1}
$$

where $G_{0} \equiv g_{0}+G_{22} E\left(P\left(e_{t+1}\right)\right)$, while

$$
u_{t+1} \equiv G_{2} \varepsilon_{t+1}+G_{12}\left(\omega_{t}^{(1)} \otimes \varepsilon_{t+1}\right)+G_{22}\left[P\left(\varepsilon_{t+1}\right)-E\left(P\left(\varepsilon_{t+1}\right)\right)\right]
$$

is a serially uncorrelated, mean zero, disturbance. The (conditional) variance of $u_{t+1}$ can easily be computed (see below). Note that $u_{t+1}$ is non-Gaussian, as $u_{t+1}$ depends on squares and cross-products of the elements of $\varepsilon_{t+1}$, and on the product of $\varepsilon_{t+1}$ and $\omega_{t}^{(1)}$. (The absence of serial correlation of $u_{t+1}$ follows from the assumption that $\varepsilon_{t+1}$ is serially independent.)

Importantly, the state equation (6) is linear in the augmented state vector $Z_{t}$ consisting of the second- and first-order accurate variables, and of the squares and crossproducts of first-order accurate variables. ${ }^{6}$ This allows convenient closed-form determination of the one-period-ahead conditional mean and variance of the state vector (see below).

\subsection{Observation Equation}

At $t=1, \ldots, T$, the analyst observes a vector $y_{t}$ of $n_{y}$ variables that are linear functions of the state vector $\omega_{t}$ plus i.i.d. measurement error that is independent of

\footnotetext{
6 Aruoba et al. (2012) estimate a pruned univariate quadratic time series model, using particle filter methods. These authors discard the term that is quadratic in $\varepsilon_{t+1}$ on the right-hand side of (4). By contrast, the paper here allows for non-zero coefficients on second-order terms in $\varepsilon_{t+1}$, and it develops a deterministic filter that can be applied to multivariate models.
} 
the state vector, at all leads and lags: $y_{t}=\gamma \omega_{t}+\psi_{t}$ where $\gamma$ is an $n_{y} \mathrm{x} n$ matrix and $\psi_{t} \sim N\left(0, \Sigma_{\psi}\right)$ is an $n_{y} \mathrm{x} 1$ vector of measurement errors; $\Sigma_{\psi}$ is a diagonal matrix. The observation equation can be written as:

$$
y_{t}=\Gamma Z_{t}+\psi_{t}, \text { with } \Gamma \equiv(\gamma, 0)
$$

\subsection{The Filter}

Let $\Upsilon^{t} \equiv\left\{y_{\tau}\right\}_{\tau=1}^{t}$ be the observables known at date $t ; X_{t, \tau} \equiv \mathrm{E}\left(X_{t} \mid \Upsilon^{\tau}\right)$ and $V_{t, \tau}^{X} \equiv$ $E\left(\left[X_{t}-X_{t, \tau}\right]\left[X_{t}-X_{t, \tau}\right]^{\prime} \mid \Upsilon^{\tau}\right)$ denote the mean and variance of the column vector $X_{t}$, given $\Upsilon^{\tau}$. The unconditional mean and variance are denoted by $E\left(X_{t}\right)$ and $V\left(X_{t}\right)$.

Given $Z_{t, t}$ and $V_{t, t}^{Z}$, the $1^{\text {st }}$ and $2^{\text {nd }}$ moments of the augmented state vector $Z_{t}$ conditional on $\Upsilon^{t}$, we can compute one-period-ahead conditional moments of $Z_{t+1}$ using (6):

$$
\begin{gathered}
Z_{t+1, t}=G_{0}+G_{1} Z_{t, t}, \\
V_{t+1, t}^{Z}=G_{1} V_{t, t}^{Z} G_{1}^{\prime}+V_{t+1, t}^{u}, \text { with } \\
V_{t+1, t}^{u} \equiv G_{2} V_{\varepsilon} G_{2}^{\prime}+G_{12}\left(\omega_{t, t}^{(1)} \otimes \Sigma_{\varepsilon}\right) G_{2}^{\prime}+G_{2}\left(\omega_{t, t}^{(1)} \otimes \Sigma_{\varepsilon}\right)^{\prime} G_{12}^{\prime} \\
+G_{12}\left\{\left(V_{t, t}^{\omega^{(1)}}+\omega_{t, t}^{(1)} \omega_{t, t}^{(1) \prime}\right) \otimes \Sigma_{\varepsilon}\right\} G_{12}^{\prime}+G_{22} V\left(P\left(\varepsilon_{t+1}\right)\right) G_{22}^{\prime}
\end{gathered}
$$

(see Appendix). Here and henceforth the parameter $\xi$ that indexes the size of the exogenous shocks is normalized at $\xi=1$

To generate $Z_{t+1, t+1}, V_{t+1, t+1}^{Z}$, I apply the linear updating equation of the standard Kalman filter (e.g., Hamilton 1994, ch. 13) to the state-space representation (6), (7): ${ }^{7}$

$$
\begin{aligned}
Z_{t+1, t+1} & =Z_{t+1, t}+\phi_{t} \cdot\left(y_{t+1}-y_{t+1, t}\right), \text { with } y_{t+1, t}=\Gamma Z_{t+1, t}, \\
\text { and } \phi_{t} & =V_{t+1, t}^{Z} \Gamma^{\prime}\left\{\Gamma V_{t+1, t}^{Z} \Gamma^{\prime}+\Sigma_{\psi}\right\}^{-1}, \\
V_{t+1, t+1}^{Z} & =V_{t+1, t}^{Z}-V_{t+1, t}^{Z} \Gamma^{\prime}\left\{\Gamma V_{t+1, t}^{Z} \Gamma^{\prime}+\Sigma_{\psi}\right\}^{-1} \Gamma V_{t+1, t}^{Z} .
\end{aligned}
$$

The filter is started with the unconditional mean and variance of $Z_{0}: Z_{0,0}=E\left(Z_{0}\right)$, $V_{0,0}^{Z} \equiv V\left(Z_{0}\right) ; \quad Z_{t+1, t+1}$ and $V_{t+1, t+1}^{Z}$ for $t \geq 0$ are computed by iterating on (8)-(12). ${ }^{8}$ Henceforth, I refer to this filter as the 'KalmanQ' filter. Computer code that implements KalmanQ is available from the author.

$E\left(Z_{0}\right)$ and $V\left(Z_{0}\right)$ can be computed exactly; see the Appendix. The linear updating formula (11) would be an exact algorithm for computing the conditional expecta-

\footnotetext{
${ }^{7}$ Linear updating rules are likewise assumed by Andreasen (2012) and Ivashchenko (2014) who also develop deterministic filters for second-order approximated DSGE models (see above).

${ }^{8}$ It is assumed that the inverse of $\Gamma V_{t+1, t}^{Z} \Gamma+\Sigma_{\psi}$ (covariance matrix of prediction errors of observables) exists. A sufficient condition for this is that $\Sigma_{\psi}$ is positive definite, as assumed in the numerical experiments below.
} 
tion $Z_{t+1, t+1}$, if $Z_{t+1}$ and the observables were (jointly) Gaussian, as then $Z_{t+1, t+1}$ would be a linear function of the data. This condition is not met in the secondorder approximated model, as the disturbance $u_{t+1}$ of the state equation (6) is non-Gaussian. However, as shown below, the KalmanQ filter closely tracks the true latent states. ${ }^{9}$ Without Gaussianity, $Z_{t+1, t+1}$ is a non-linear function of data $\Upsilon^{t+1}: Z_{t+1, t+1}=\phi\left(y_{t+1}, \Upsilon^{t}\right)$. (11) can be viewed as a linear approximation of this function: $Z_{t+1, t+1} \cong \phi_{t} \cdot\left(y_{t+1}-y\right)$, where $y$ is the steady state of $y_{t+1}$ and $\phi_{t} \equiv \partial \phi\left(y_{t+1}, \Upsilon^{t}\right) /\left.\partial y_{t+1}\right|_{y_{t+1}=y}$. By the Law of Iterated Expectations, $Z_{t+1, t}=E\left(Z_{t+1, t+1} \mid \Upsilon^{t}\right)$, and thus: $Z_{t+1, t+1}-Z_{t+1, t} \cong \phi_{t} \cdot\left(y_{t+1}-y_{t+1, t}\right)$.

When the linearized model is the true DGP (i.e. when $F_{0}=0, F_{11}=0, F_{12}=0$, $F_{22}=0$ ), then the filter here is identical to the conventional linear Kalman filter, and the updating formula (11) holds exactly. In the presence of second-order model terms, KalmanQ is more accurate than a conventional Kalman filter that assumes that the linearized model (3) is the true DGP; see below.

\subsection{Quasi-Maximum Likelihood Estimation of Model Parameters}

If model parameters are unknown, then a quasi-maximum likelihood (QML) estimate of those parameters can be obtained by maximizing the function $L\left(\Upsilon^{T} \mid \theta\right) \equiv$ $\sum_{t=1}^{T} \ln h\left(y_{t} \mid y_{t, t-1}(\theta) ; V_{t, t-1}^{y}(\theta)\right)$, with respect to the vector of unknown parameters, $\theta$. Here $h(y \mid \mu ; V)$ is the multivariate normal density with mean $\mu$ and variance $V$. For a given $\theta, \quad y_{t, t-1}(\theta) \equiv \Gamma Z_{t, t-1}(\theta)$ is the prediction of $y_{t}$ generated by KalmanQ, based on date $t-1$ information, $\Upsilon^{t-1} ; \quad V_{t, t-1}^{y}(\theta) \equiv \Gamma V_{t, t-1}^{Z}(\theta) \Gamma^{\prime}+\Sigma_{\psi}$ is the conditional variance of $y_{t}$, given $\Upsilon^{t-1}$. Under conditions discussed in Hamilton (1994, ch. 13), the QML estimator $\theta_{T}^{Q M L}$ is asymptotically normal: $\sqrt{T}\left(\theta_{T}^{Q M L}-\theta_{0}\right) \rightarrow N\left(0,\left(J_{2}\left(J_{1}\right)^{-1} J_{2}\right)^{-1}\right)$, where $\theta_{0}$ is the true parameter vector and $J_{1} \equiv \operatorname{plim} T^{-1} \sum_{t=1}^{T} \eta_{t}\left(\theta_{0}\right) \eta_{t}\left(\theta_{0}\right)^{\prime}$, with $\eta_{t}\left(\theta_{0}\right) \equiv \partial \log \left(h_{t}\left(\theta_{0}\right)\right) / \partial \theta$, $h_{t}(\theta) \equiv h\left(y_{t} \mid y_{t, t-1}\left(\theta_{0}\right) ; V_{t, t-1}^{z}\left(\theta_{0}\right)\right)$ and $J_{2} \equiv \operatorname{plim} T^{-1} \sum_{t=1}^{T} \partial^{2} \log \left(h_{t}\left(\theta_{0}\right)\right) / \partial \theta \partial \theta^{\prime}$.

\subsection{Ivashchenko’s (2014) Quadratic Kalman Filter (QKF)}

The QKF posits that the unpruned second-order approximated model (2) is the true DGP [instead of the pruned system (3), (4)]. The QKF is derived under the assumption that the vector of estimation errors of filtered states and exogenous innovations $\Theta_{t, t+1} \equiv\left(\omega_{t}^{\prime}-E_{t} \omega_{t}^{\prime}, \varepsilon_{t+1}^{\prime}\right)^{\prime}$ is Gaussian. Ivashchenko (2014) assumes a linear updating rule similar to (11): $\omega_{t+1, t+1}=\omega_{t+1, t}+\vartheta_{t} \cdot\left(y_{t+1}-y_{t+1, t}\right)$, where $\vartheta_{t}$ is defined analogously to $\phi_{t}$ in (12); thus knowledge of $V_{t+1, t}^{\omega}$ (one-period ahead conditional variance of $\omega_{t+1}$ ), is required for the QKF filter. (2) implies that $V_{t+1, t}^{\omega}$ depends on the conditional fourth moments of $\Theta_{t+1}$. Under the assumed normality of $\Theta_{t+1}$, it is easy to compute those fourth moments in closed-form, as functions of the conditional variance of $\Theta_{t+1}$.

\footnotetext{
${ }^{9}$ Recall that the observable $y_{t+1}$ is a linear function of $Z_{t+1}$ [see (7)]; this may help to explain the good performance of the linear updating rule.
} 


\section{Monte Carlo Evidence}

\subsection{A Textbook RBC Model}

The method is tested for a basic RBC model. Assume a representative infinitelylived household whose date $t$ expected lifetime utility $V_{t}$ is given by $V_{t}=\left\{\frac{1}{1-\sigma} C_{t}^{1-\sigma}-\frac{1}{1+1 / \eta} N_{t}^{1+1 / \eta}\right\}+\lambda_{t} \beta E_{t} V_{t+1}$, where $C_{t}$ and $N_{t}$ are consumption and hours worked, at $t$, respectively. $\sigma>0$ and $\eta>0$ are the risk aversion coefficient and the (Frisch) labor supply elasticity. $\lambda_{t}$ is an exogenous random taste (discount factor) shock that equals unity in steady state. $0<\beta<1$ is the steady state subjective discount factor. The household maximizes expected lifetime utility subject to the period $t$ resource constraint

$$
C_{t}+I_{t}=Y_{t}
$$

where $Y_{t}$ and $I_{t}$ are output and gross investment, respectively. The production function is

$$
Y_{t}=\theta_{t} K_{t}^{\alpha} N_{t}^{1-\alpha}
$$

where $K_{t}$ is the beginning-of-period $t$ capital stock, and $\theta_{t}>0$ is exogenous total factor productivity (TFP). The law of motion of the capital stock is

$$
K_{t+1}=(1-\delta) K_{t}+I_{t}
$$

$0<\alpha, \delta<1$ are the capital share and the capital depreciation rate, respectively. The household's first-order conditions are:

$$
\lambda_{t} E_{t} \beta\left(C_{t+1} / C_{t}\right)^{-\sigma}\left(\theta_{t+1} \alpha K_{t+1}^{\alpha-1} N_{t+1}^{1-\alpha}+1-\delta\right)=1, \quad C_{t}^{-\sigma}(1-\alpha) \theta_{t} K_{t}^{\alpha} N_{t}^{-\alpha}=N_{t}^{1 / \eta} .
$$

The forcing variables follow independent autoregressive processes:

$$
\ln \left(\theta_{t}\right)=\rho_{\theta} \ln \left(\theta_{t-1}\right)+\varepsilon_{\theta, t}, \quad \ln \left(\lambda_{t}\right)=\rho_{\lambda} \ln \left(\lambda_{t-1}\right)+\varepsilon_{\lambda, t}, 0<\rho_{\theta}, \rho_{\lambda}<1,
$$

where $\varepsilon_{\theta, t}$ and $\varepsilon_{\lambda, t}$ are normal i.i.d. white noises with standard deviations $\sigma_{\theta}$ and $\sigma_{\lambda}$.

The numerical simulations discussed below assume $\beta=0.99, \eta=4, \alpha=0.3$, $\delta=0.025, \rho_{\theta}=\rho_{\lambda}=0.99$; parameter values in that range are standard in quarterly macro models. The risk aversion coefficient is set at a high value, $\sigma=10$, so that the model has enough curvature to allow for non-negligible differences between the second-order accurate and linear model approximations. One model variant assumes shocks that are much larger than the shocks in standard macro models, in order to generate big differences between the two approximations: $\sigma_{\theta}=0.20, \quad \sigma_{\lambda}=0.01$. I refer to this variant as the 'big shocks' variant. I also consider a second 'small shocks' variant, in which the standard deviations of shocks are twenty times smaller: 
$\sigma_{\theta}=0.01, \sigma_{\lambda}=0.0005$ (conventional RBC models assume that the standard deviation of TFP innovations is about $1 \%$; e.g., Kollmann 1996). ${ }^{10}$ The observables are assumed to be GDP, consumption, investment and hours worked; independent measurement error is added to log observables. Measurement error has a standard deviation of 0.04 (0.002) for each observable, in the model variant with big (small) shocks.

Chris Sims' MATLAB program gensys 2 is used to compute first- and second-order accurate model solutions. The model is approximated in terms of logged variables. I apply the gensys2 algorithm to the 7-equations system (13)-(17) using the state vector $\omega_{t} \equiv\left(\ln \left(K_{t+1}\right), \ln \left(Y_{t}\right), \ln \left(C_{t}\right), \ln \left(I_{t}\right), \ln \left(N_{t}\right), \ln \left(\theta_{t}\right), \ln \left(\lambda_{t}\right)\right)$. Simplifying the system (e.g. by plugging (14) and (15) into (13) and omitting $Y_{t}, I_{t}$ ) does not affect the results.

\subsubsection{Predicted Standard Deviations}

Table 1 reports unconditional standard deviations of 7 logged variables (GDP, consumption, investment, capital, hours, TFP and the taste shock $\lambda$ ) generated by the firstand second-order approximations. ${ }^{11}$ Model variants with both shocks, and variants with just one type of shock, are considered; moments for non-HP (Hodrick-Prescott) filtered variables are shown, as well as moments of HP filtered variables (smoothing parameter: 1600).

In the 'big shocks' model variant, the standard deviations of endogenous variables are huge; e.g., with both shocks, the standard deviation of (non-HP filtered) GDP is $176 \%$ (82\%) under the second-order (first-order) approximation; GDP is thus about twice as volatile under the second-order approximation (than in the linearized model). ${ }^{12}$ The capital stock, investment and hours worked (non-HP filtered) are about one-half more volatile under the second-order approximation than under the linear approximation. By contrast, consumption volatility is similar across the two approximations. Consumption is much less volatile than GDP, due to the assumed high risk aversion of the household. The preference shock $(\lambda)$ is the main source of fluctuations in the capital stock, GDP and investment; the TFP shock $(\theta)$ is the main driver of consumption. The correlation between the second- and first-order approximations of a given variable is noticeably below unity, in the model variant with big shocks: e.g., about 0.7 for capital and investment, and 0.5 for GDP.

The 'small shocks' model variant generates much smaller standard deviations of endogenous variables that are roughly in line with predicted moments reported in the RBC literature (e.g., Kollmann 1996); e.g., the predicted standard deviation of HP-filtered GDP and investment are about 1 and $5 \%$, respectively (with both

\footnotetext{
10 The relative size of the TFP and taste shocks assumed here (i.e. $\sigma_{\theta}$ 20-times larger than $\sigma_{\psi}$ ) ensures that each shock accounts for a non-negligible share of the variance of the endogenous variables; see below.

11 The statistics in Table 1 are shown for variables without measurement error. The ranking of volatilities generated by the two approximations and shocks is not affected by the presence of measurement error.

12 HP filtered variables are markedly less volatile than non-HP filtered variables; however, volatility remains much higher under the second-order approximation than under the linear approximation, in the 'big shocks' variant. E.g. the standard dev. of HP filtered GDP is $47 \%$ (23\%) under the second- (first-) order approximation.
} 
Table 1 RBC model: predicted standard deviations

\begin{tabular}{llllllll}
\hline$Y$ & $C$ & $I$ & $K$ & $N$ & $\theta$ \\
\hline$(1)$ & $(2)$ & $(3)$ & $(4)$ & $(5)$ & $(6)$ \\
\hline
\end{tabular}

(a) Model variant with big shocks $\left(\sigma_{\theta}=0.20, \sigma_{\lambda}=0.01\right)$

(a.1) Non-HP filtered variables

Second-order model approximation

$\begin{array}{cccccccc}\text { Both shocks } & 1.757 & 0.300 & 5.366 & 3.400 & 2.609 & 1.418 & 0.071 \\ \text { Just } \theta \text { shock } & 0.492 & 0.264 & 1.387 & 0.959 & 1.761 & 1.418 & 0.000 \\ \text { Just } \lambda \text { shock } & 1.558 & 0.133 & 4.799 & 3.096 & 1.762 & 0.000 & 0.071 \\ \text { Linearized model } & & & & & & & \\ \text { Both shocks } & 0.817 & 0.276 & 3.269 & 2.364 & 1.862 & 1.418 & 0.071 \\ \text { Just } \theta \text { shock } & 0.469 & 0.264 & 1.285 & 0.929 & 1.751 & 1.418 & 0.000 \\ \text { Just } \lambda \text { shock } & 0.669 & 0.083 & 3.006 & 2.174 & 0.634 & 0.000 & 0.071\end{array}$

(a.2) HP filtered variables

Second-order model approximation

$\begin{array}{cccccccc}\text { Both shocks } & 0.469 & 0.053 & 1.962 & 0.115 & 0.688 & 0.259 & 0.013 \\ \text { Just } \theta \text { shock } & 0.124 & 0.037 & 0.483 & 0.038 & 0.212 & 0.259 & 0.000 \\ \begin{array}{c}\text { Just } \lambda \text { shock } \\ \text { Linearized model }\end{array} & 0.420 & 0.034 & 1.706 & 0.104 & 0.608 & 0.000 & 0.013 \\ \text { Both shocks } & 0.229 & 0.041 & 1.059 & 0.095 & 0.350 & 0.259 & 0.013 \\ \text { Just } \theta \text { shock } & 0.118 & 0.037 & 0.416 & 0.037 & 0.205 & 0.259 & 0.000 \\ \text { Just } \lambda \text { shock } & 0.196 & 0.016 & 0.974 & 0.087 & 0.284 & 0.000 & 0.013\end{array}$

(b) Model variant with small shocks $\left(\sigma_{\theta}=0.01, \sigma_{\lambda}=0.0005\right)$

(b.1) Non-HP filtered variables

Second-order model approximation

$\begin{array}{llllllll}\text { Both shocks } & 0.041 & 0.014 & 0.164 & 0.118 & 0.093 & 0.071 & 0.004 \\ \text { Just } \theta \text { shock } & 0.023 & 0.013 & 0.064 & 0.046 & 0.088 & 0.071 & 0.000 \\ \text { Just } \lambda \text { shock } & 0.034 & 0.004 & 0.151 & 0.109 & 0.032 & 0.000 & 0.004 \\ \text { Linearized model } & & & & & & & \\ \text { Both shocks } & 0.041 & 0.014 & 0.163 & 0.118 & 0.093 & 0.071 & 0.004 \\ \text { Just } \theta \text { shock } & 0.023 & 0.013 & 0.064 & 0.046 & 0.088 & 0.071 & 0.000 \\ \text { Just } \lambda \text { shock } & 0.033 & 0.004 & 0.150 & 0.109 & 0.032 & 0.000 & 0.004\end{array}$

(b.2) HP filtered variables

Second-order model approximation

\begin{tabular}{llllllll} 
Both shocks & 0.011 & 0.002 & 0.053 & 0.005 & 0.018 & 0.013 & 0.001 \\
Just $\theta$ shock & 0.006 & 0.002 & 0.021 & 0.002 & 0.010 & 0.013 & 0.000 \\
Just $\lambda$ shock & 0.010 & 0.001 & 0.049 & 0.004 & 0.014 & 0.000 & 0.001 \\
$\begin{array}{c}\text { Linearized model } \\
\text { Both shocks }\end{array}$ & 0.011 & 0.002 & 0.053 & 0.005 & 0.018 & 0.013 & 0.001 \\
Just $\theta$ shock & 0.006 & 0.002 & 0.021 & 0.002 & 0.010 & 0.013 & 0.000 \\
Just $\lambda$ shock & 0.010 & 0.001 & 0.049 & 0.004 & 0.014 & 0.000 & 0.001 \\
\hline
\end{tabular}

Note: Standard deviations (SD) of logged variables (listed above Cols. (1)-(7)), without measurement error, are shown for the RBC model in Sect. 3.1. The SD were computed using the formulae in the Appendix. SD are not reported in \%.

Panel (a) ('Big shocks') assumes SD of innovations to TFP $\theta$ and to the taste parameter $\lambda$ of 20 and $1 \%$, respectively. Panel (b) ('Small shocks') sets these SD at 1 and $0.05 \%$, respectively. Rows labeled 'Both shocks'; 'Just $\theta$ shock'; and 'Just $\lambda$ shock' show moments predicted with simultaneous two shocks; with just the TFP shock; and with just the taste shock, respectively. Panels (a.1) and (b.1) report moments of Non-HP filtered variables; Panels (a.2) and (b.2) pertain to HP filtered variables (smoothing parameter: 1600). $Y$ : GDP; $C$ : consumption; $I$ : gross investment; $K$ : capital stock; $N$ : hours worked 
shocks). With small shocks, it remains true that variables are more volatile in the second-order model than in the linearized model, however, the difference is barely noticeable. E.g., the ratio of the GDP [investment] standard dev. across the $2^{\text {nd }} / 1^{\text {st }}$ order approximations is merely 1.005 [1.002].

\subsubsection{Filter Accuracy}

I generate 50 simulation runs of $T=500$ and of $T=100$ periods for the observables, using the second-order (pruned) state equation of the RBC model. Each run is initialized at the unconditional mean of the state vector. I apply the KalmanQ filter to the simulated observables (with measurement error). I also use Ivashchenko's (2014) Quadratic Kalman Filter 'QKF' and a conventional Kalman filter, referred to as 'KalmanL' (that treats the linearized model (3) as the true DGP). In addition, the standard particle filter (as described in An and Schorfheide 2007)—referred to a ' $\mathrm{PF}(p)$ ', where $p$ is the number of particles-is applied to the pruned state equation (4); for the simulation runs with $\mathrm{T}=500$ periods, 100,000 particles are employed; for runs with $\mathrm{T}=100$ periods, versions of the PF with 100,000 and with 500,000 particles are used. ${ }^{13}$ Accuracy is evaluated for the 7 logged latent variables considered in Table 1.

In each simulation run $s=1, \ldots, 50$, the root mean square error (RMSE) is computed, across all (logged) 7 variables, $R M S E_{s, A l l} \equiv\left(\frac{1}{7 T} \sum_{i=1}^{7} \sum_{t=1}^{T}\left(\omega_{s, t}^{i}-\omega_{s, t, t}^{i}\right)^{2}\right)^{1 / 2}$, and separately for each individual variable $i=1, \ldots 7, \quad R M S E_{s, i} \equiv\left(\frac{1}{\mathrm{~T}} \Sigma_{t=1}^{T}\left(\omega_{s, t}^{i}-\omega_{s, t, t}^{i}\right)^{2}\right)^{1 / 2}$, where $\omega_{s, t}^{i}$ is the true date $t$ value of variable $i$ in run $s$, while $\omega_{s, t, t}^{i}$ is the filtered estimate (conditional expectation) of that variable, given the date $t$ information set. Table 2 reports RMSEs that are averaged across simulation runs. In the Panels labeled 'Average RMSEs', Column (1) shows average RMSE, across all 7 variables, $\frac{1}{50} \sum_{s=1}^{50} R M S E_{S, A L L}$, while Cols. (2)-(8) separately show average RMSEs for each individual variable $i, \frac{1}{50} \sum_{s=1}^{50} R M S E_{S, i}$. Also reported are maximum estimation errors across all variables, periods and runs, as well as maximum estimation errors for each variable $i$ (across all periods and all simulation runs); see Panels labeled 'Maximum errors'. These accuracy measures are reported for each of the filters (see rows labeled 'KalmanQ', 'QKF', 'PF(100,000)', 'PF(500,000)', and 'KalmanL'). In addition, I report the fraction of simulation runs in which the KalmanQ filter generates lower RMSEs and lower maximum estimation error than the other filters.

Table 2 shows that the KalmanQ filter is more accurate than the PF and KalmanL filters, in all (or almost all) simulation runs - this holds for both the 'big shocks' and 'small shocks' model variants. ${ }^{14}$ In all 50 simulation runs for the 'big shocks' model

\footnotetext{
13 I apply KalmanL to de-meaned series, as the linearized model implies that the unconditional mean of state variables, expressed as differences from steady state, is zero, while variables generated from the second-order model have a non-zero mean. The initial particles used for the particle filter are drawn from a multi-variate normal distribution whose mean and variance are set to unconditional moments of the state vector.

${ }^{14}$ I also computed median absolute errors (MAEs) for the filtered series. The results (available on request) confirm the greater accuracy of the KalmanQ filter.
} 
Table 2 RBC model: accuracy of filters

\begin{tabular}{lllllllll}
\hline All variables & $Y$ & $C$ & $I$ & $K$ & $N$ & $\theta$ & $\lambda$ \\
\hline$(1)$ & $(2)$ & $(3)$ & $(4)$ & $(5)$ & $(6)$ & $(7)$ & $(8)$ \\
\hline
\end{tabular}

(a) Model variant with big shocks $\left(\sigma_{\theta}=0.20, \sigma_{\lambda}=0.01\right)$

(a.1) 50 simulation runs with $T=500$ periods

\section{Average RMSEs}

$\begin{array}{lllllllll}\text { KalmanQ } & 0.157 & 0.038 & 0.006 & 0.040 & 0.387 & 0.039 & 0.127 & 0.022 \\ \text { QKF } & - & - & - & - & - & - & - & - \\ \begin{array}{l}\text { PF }(100,000) \\ \text { KalmanL }\end{array} & 1.189 & 1.022 & 0.188 & 0.907 & 2.193 & 1.447 & 0.873 & 0.070 \\ \begin{array}{l}\text { Maximum errors } \\ \text { KalmanQ }\end{array} & 1.939 & 1.659 & 0.184 & 1.605 & 4.183 & 0.770 & 1.143 & 0.082 \\ \text { QKF } & 3.448 & 0.209 & 0.024 & 0.164 & 3.448 & 0.166 & 1.198 & 0.236 \\ \text { PF (100,000) } & - & - & - & - & - & - & - & - \\ \text { KalmanL } & 20.188 & 15.750 & 1.927 & 10.451 & 20.188 & 19.842 & 4.236 & 0.563 \\ & 14.824 & 14.824 & 1.275 & 7.849 & 13.071 & 3.820 & 12.039 & 0.568\end{array}$

Fraction of runs in which RMSE is lower for KalmanQ than for other filters

QKF

PF (100,000)

KalmanL

1.00

$\begin{array}{llll}- & - & - & - \\ 1.00 & 1.00 & 1.00 & 1.00 \\ 1.00 & 1.00 & 1.00 & 1.00\end{array}$

-
1.00

1.00

1.00

1.00

1.00

ters

\section{QKF}

PF (100,000)

$$
1.00
$$

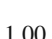

$1.00 \quad 1.00$

1.00

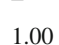

1.00

1.00
1.00

1.00
1.00 1.00
1.00

1.00
0.98

0.96

(a.2) 50 simulation runs with $T=100$ periods

\section{Average RMSEs}

$\begin{array}{lllllllll}\text { KalmanQ } & 0.176 & 0.039 & 0.006 & 0.039 & 0.435 & 0.039 & 0.141 & 0.023 \\ \text { QKF } & 6.994 & 0.142 & 0.025 & 0.065 & 17.386 & 0.130 & 2.383 & 4.962 \\ \text { PF }(100,000) & 0.828 & 0.755 & 0.121 & 0.744 & 1.370 & 0.892 & 0.679 & 0.049 \\ \text { PF (500,000) } & 0.597 & 0.527 & 0.108 & 0.499 & 0.892 & 0.695 & 0.658 & 0.030 \\ \text { KalmanL } & 1.917 & 1.448 & 0.176 & 2.063 & 3.955 & 0.973 & 0.975 & 0.067 \\ \text { Maximum errors } & & & & & & & & \\ \text { KalmanQ } & 2.855 & 0.160 & 0.023 & 0.134 & 2.855 & 0.163 & 0.901 & 0.226 \\ \text { QKF } & 1438.080 & 8.138 & 1.500 & 5.763 & 1438.080 & 5.482 & 153.500 & 606.064 \\ \text { PF (100,000) } & 13.298 & 10.615 & 1.364 & 9.944 & 13.190 & 13.298 & 2.846 & 0.414 \\ \text { PF (500,000) } & 8.018 & 4.548 & 0.560 & 8.018 & 5.680 & 4.577 & 3.425 & 0.235 \\ \text { KalmanL } & 11.866 & 9.826 & 0.655 & 8.399 & 11.866 & 3.484 & 5.093 & 0.332\end{array}$

Fraction of runs in which RMSE is lower for KalmanQ than for other filters

$\begin{array}{lllllllll}\text { QKF } & 1.00 & 1.00 & 1.00 & 0.75 & 1.00 & 0.89 & 1.00 & 1.00 \\ \text { PF }(100,000) & 1.00 & 1.00 & 1.00 & 1.00 & 0.84 & 1.00 & 1.00 & 0.64 \\ \text { PF }(500,000) & 1.00 & 1.00 & 1.00 & 1.00 & 0.84 & 1.00 & 1.00 & 0.68 \\ \text { KalmanL } & 1.00 & 1.00 & 1.00 & 1.00 & 1.00 & 1.00 & 1.00 & 0.98\end{array}$

Fraction of runs in which maximum error is lower for KalmanQ than for other filters

\begin{tabular}{lllllllll} 
QKF & 1.00 & 1.00 & 0.93 & 0.64 & 1.00 & 0.68 & 1.00 & 1.00 \\
PF $(100,000)$ & 0.96 & 1.00 & 1.00 & 1.00 & 0.84 & 1.00 & 1.00 & 0.80 \\
PF (500,000) & 1.00 & 1.00 & 1.00 & 1.00 & 0.80 & 1.00 & 1.00 & 0.76 \\
KalmanL & 1.00 & 1.00 & 1.00 & 1.00 & 1.00 & 1.00 & 1.00 & 0.96 \\
\hline
\end{tabular}


Table 2 continued

\begin{tabular}{llllllll}
\hline All variables & $Y$ & $C$ & $I$ & $K$ & $N$ & $\theta$ & $\lambda$ \\
\hline$(1)$ & $(2)$ & $(3)$ & $(4)$ & $(5)$ & $(6)$ & $(7)$ & $(8)$ \\
\hline
\end{tabular}

(b) Model variant with small shocks $\left(\sigma_{\theta}=0.01, \sigma_{\lambda}=0.0005\right)$

(b.1) 50 simulation runs with $T=500$ periods

\section{Average RMSEs}

$\begin{array}{lllllllll}\text { KalmanQ } & 0.0022 & 0.0007 & 0.0003 & 0.0019 & 0.0044 & 0.0019 & 0.0022 & 0.0001 \\ \text { QKF } & 0.0024 & 0.0007 & 0.0003 & 0.0019 & 0.0049 & 0.0019 & 0.0023 & 0.0001 \\ \text { PF }(100,000) & 0.0222 & 0.0068 & 0.0057 & 0.0109 & 0.0180 & 0.0405 & 0.0343 & 0.0007 \\ \begin{array}{l}\text { KalmanL } \\ \text { Maximum errors }\end{array} & 0.0411 & 0.0163 & 0.0059 & 0.0583 & 0.0585 & 0.0397 & 0.0312 & 0.0018 \\ \text { KalmanQ } & 0.0475 & 0.0041 & 0.0011 & 0.0074 & 0.0475 & 0.0081 & 0.0163 & 0.0012 \\ \text { QKF } & 0.0601 & 0.0103 & 0.0011 & 0.0078 & 0.0601 & 0.0080 & 0.0216 & 0.0017 \\ \text { PF }(100,000) & 0.2437 & 0.0434 & 0.0330 & 0.0723 & 0.2438 & 0.2295 & 0.1939 & 0.0054 \\ \text { KalmanL } & 0.2293 & 0.0753 & 0.0248 & 0.2146 & 0.2294 & 0.1837 & 0.1512 & 0.0074\end{array}$

Fraction of runs in which RMSE is lower for KalmanQ than for other filters

$\begin{array}{lllllllll}\text { QKF } & 0.60 & 0.32 & 0.34 & 0.44 & 0.58 & 0.54 & 0.60 & 0.64 \\ \text { PF (100,000) } & 1.00 & 1.00 & 1.00 & 1.00 & 0.98 & 1.00 & 1.00 & 1.00 \\ \text { KalmanL } & 1.00 & 1.00 & 1.00 & 1.00 & 1.00 & 1.00 & 1.00 & 1.00\end{array}$

Fraction of runs in which maximum error is lower for KalmanQ than for other filters

$\begin{array}{lllllllll}\text { QKF } & 0.54 & 0.60 & 0.50 & 0.52 & 0.54 & 0.46 & 0.64 & 0.56 \\ \text { PF (100,000) } & 1.00 & 1.00 & 1.00 & 1.00 & 1.00 & 1.00 & 1.00 & 1.00 \\ \text { KalmanL } & 0.98 & 1.00 & 1.00 & 0.98 & 0.92 & 1.00 & 0.98 & 0.94\end{array}$

(b.2) 50 simulation runs with $T=100$ periods

Average RMSEs

\begin{tabular}{lllllllll} 
KalmanQ & 0.0042 & 0.0007 & 0.0003 & 0.0019 & 0.0099 & 0.0019 & 0.0035 & 0.0002 \\
QKF & 0.0049 & 0.0007 & 0.0003 & 0.0019 & 0.0121 & 0.0019 & 0.0041 & 0.0003 \\
PF (100,000) & 0.0244 & 0.0060 & 0.0051 & 0.0096 & 0.0368 & 0.0362 & 0.0313 & 0.0009 \\
PF (500,000) & 0.0223 & 0.0046 & 0.0039 & 0.0074 & 0.0398 & 0.0278 & 0.0271 & 0.0010 \\
KalmanL & 0.0508 & 0.0224 & 0.0078 & 0.0819 & 0.0716 & 0.0466 & 0.0357 & 0.0019 \\
Maximum errors & & & & & & & \\
KalmanQ & 0.0496 & 0.0033 & 0.0009 & 0.0073 & 0.0496 & 0.0068 & 0.0171 & 0.0012 \\
QKF & 0.0543 & 0.0048 & 0.0009 & 0.0069 & 0.0543 & 0.0069 & 0.0193 & 0.0015 \\
PF (100,000) & 0.1866 & 0.0319 & 0.0261 & 0.0551 & 0.1866 & 0.1848 & 0.1473 & 0.0046 \\
PF (500,000) & 0.2591 & 0.0224 & 0.0171 & 0.0402 & 0.2591 & 0.1189 & 0.1143 & 0.0065 \\
KalmanL & 0.3001 & 0.0918 & 0.0263 & 0.3001 & 0.2900 & 0.1846 & 0.1424 & 0.0083 \\
Fraction of runs in which RMSE is lower for KalmanQ than for other filters & & & \\
QKF & 0.66 & 0.12 & 0.26 & 0.46 & 0.66 & 0.48 & 0.66 & 0.66 \\
PF (100,000) & 1.00 & 1.00 & 1.00 & 1.00 & 0.94 & 1.00 & 1.00 & 0.98 \\
PF (500,000) & 1.00 & 1.00 & 1.00 & 1.00 & 0.90 & 1.00 & 1.00 & 0.92 \\
KalmanL & 1.00 & 1.00 & 1.00 & 1.00 & 0.94 & 1.00 & 1.00 & 0.90 \\
Fraction of runs in which maximum error is lower for KalmanQ than for other filters & & & 0.56 \\
QKF & 0.64 & 0.24 & 0.46 & 0.60 & 0.64 & 0.48 & 0.56 & 0.66 \\
PF (100,000) & 1.00 & 1.00 & 1.00 & 1.00 & 1.00 & 1.00 & 1.00 & 1.00 \\
PF (500,000) & 1.00 & 1.00 & 1.00 & 1.00 & 0.92 & 1.00 & 1.00 & 0.96 \\
KalmanL & 0.98 & 1.00 & 1.00 & 1.00 & 0.94 & 1.00 & 1.00 & 0.90 \\
\hline
\end{tabular}


Table 2 continued

Note: The Table reports average Root Mean Squared Errors (RMSEs) and maximum estimation errors of estimated latent states, across simulation runs (errors are not expressed in \%), for different variants of the RBC model; accuracy is reported across all logged variables (Col. (1) 'All variables'), and separately for each logged variable (see Cols. (2)-(8) labeled ' $Y$ ', ..., ' $\lambda$ '); see Sect. 3.1. for further information. KalmanQ: the filter for pruned second-order models developed in this paper; $Q K F$ : Quadratic Kalman Filter (Ivashchenko 2014) that assumes that the unpruned second-order model (2) is the true DGP; PF:(p) standard particle filter with $p$ particles; KalmanL: standard Kalman filter that assumes that the linearized DSGE model is the true DGP.

Panel (a) ('Big shocks') assumes std. of TFP and taste shock innovations of 20 and $1 \%$, respectively; panel (b) ('Small shocks') sets these standard deviations at 1 and $0.05 \%$, respectively. $Y$ : GDP; $C$ : consumption; $I$ : gross investment; $K$ : capital stock; $N$ : hours worked; $\theta$ : TFP; $\lambda$ : taste shock (all variables are expressed in logs).

The time paths for filtered state estimates generated by the QKF exploded in all 50 simulation runs of the 'Big shocks' model variant with $\mathrm{T}=500$ periods; see Panel (a.1). Thus, no RMSEs and Maximum Errors are reported for the QKF in Panel (a.1). The QKF exploded in $44 \%$ of the 50 simulation runs of the 'Big shocks' model variant with $\mathrm{T}=100$ periods; see Panel (a.2). The RMSEs and Maximum Errors for the QKF reported in Panel (a.2) pertain to the $56 \%$ of the simulation runs in which the QKF did not explode; the fraction of runs in which RMSEs and Maximum Errors are lower for KalmanQ than for QKF only pertains to the runs in which QKF did not explode.

The QKF did not explode in the simulation runs of the 'Small shocks' model variant (Panel (b))

variant with $\mathrm{T}=500$ periods (see Panel (a.1)), the QKF generated time paths of filtered state estimates that diverged explosively from the true states. ${ }^{15}$ That problem never arose for the KalmanQ filter. In the 'big shocks' model variant with $\mathrm{T}=100$ periods (see Panel (a.2)), the QKF exploded in 22 of the 50 simulation runs (44\%); for that variant, the average RMSEs and maximum errors reported for the QKF pertain to the simulation runs in which the QKF did not explode; in those simulation runs the KalmanQ filter is markedly more accurate than the QKF. Average RMSEs generated by KalmanQ are often orders of magnitudes smaller than the RMSEs generated by the particle filter, and that even when 500,000 particles are used. E.g., for the simulation runs of the 'big shocks' model variant with $\mathrm{T}=100$ periods, the average RMSEs for GDP are 0.039, 0.142, 0.755, 0.527 and 1.488, respectively, for KalmanQ, QKF, PF(100,000), PF(500,000), and KalmanL; the corresponding maximum errors are $0.160,8.138,10.615,4.548$ and 9.826, respectively (see Panel (a.2), Col. (2)).

In the 'small shocks' simulation runs (Table 2, Panels (b.1) and (b.2)), all the filters are more accurate than in the 'big shocks' simulations, and thus the absolute accuracy differences between the filters are smaller. The QKF did not explode in the 'small shocks' simulations. The filtered estimates of latent states generated by the KalmanQ filter and by the QKF are now broadly similar (across all variables, the KalmanQ filter is more accurate than the QKF in 54-66\% of all simulation runs; see Column (1)). For the 'small shocks' simulation runs with $\mathrm{T}=100$ periods, the average RMSEs for GDP are $0.0007,0.0007,0.0060,0.0046$

\footnotetext{
15 Once the QKF filtered estimates of the second-order accurate state variables $\omega_{t, t}$ reach large values, the one-step ahead covariance matrix $V_{t+1, t}^{\omega}$ takes huge values too (in the QKF, $V_{t+1, t}^{\omega}$ depends on $\omega_{t, t}$ ); at that point the observations are no longer able to correct the filtered series, and the filtered series may start to diverge explosively.
} 
and 0.0224, for KalmanQ, QKF, $\mathrm{PF}(100,000), \operatorname{PF}(500,000)$ and KalmanL respectively, while corresponding maximum errors are $0.0033,0.0048,0.0319,0.0224$ and 0.0918 (see Panel (b.2)). The relative improvement in accuracy from using the KalmanQ filter thus remains sizable, relative to the particle filter, and relative to KalmanL.

Note that the accuracy checks considered so far are based on pruned simulated sample paths (generated using (3),(4)). It seems interesting to also apply the filters to sample paths generated by the unpruned model (2). For the 'big shocks' model variant, all unpruned sample paths of length $\mathrm{T}=100$ and $\mathrm{T}=500$ explode. By contrast, for the 'small shocks' model variant, the unpruned sample paths fail to explode-in fact, those paths are highly correlated with the pruned sample paths; the performance of the filters is hence similar to that reported in Table 2, for the 'small shocks' pruned model variant. ${ }^{16}$ Detailed results are available on request.

\subsubsection{Computing Time}

KalmanQ, QKF, the particle filters with 100,000 and 500,000 particles and KalmanL require $0.03,0.05,14.69,81.21$ and 0.01 seconds, respectively, to filter simulated series of $\mathrm{T}=100$ periods generated by the RBC model, on a desktop computer with a 64bit operating system and a $3.4 \mathrm{Ghz}$ processor. For series of $\mathrm{T}=500$ periods, the corresponding computing times are $0.12,0.21,73.72,401.58$ and 0.04 seconds, respectively. Thus, the KalmanQ filter is about 500 (3000) times faster than the particle filter with 100,000 $(500,000)$ particles, and approximately $40 \%$ faster than the QKF.

For a sufficiently large number of particles, the particle filter is (asymptotically) an exact algorithm for computing the conditional expectation of the state vector. However, the experiments in Table 2 suggest that a very large number of particles (above 500,000) is needed to outperform KalmanQ; the computational cost of using such a large number of particles would be substantial.

\subsubsection{Evaluating the Quasi-Maximum Likelihood (QML) Parameter Estimates}

For 50 simulations runs of the 'big shocks' model variant and of the 'small shocks' variant, with $\mathrm{T}=100$ periods, I computed QML estimates of the risk aversion coefficient $(\sigma)$, the labor supply elasticity $(\eta)$, the autocorrelations of the forcing variables $\left(\rho_{\theta}, \rho_{\lambda}\right)$ and the standard deviations of the innovations to the forcing variables $\left(\sigma_{\theta}, \sigma_{\lambda}\right)$. (As before, four observables are assumed: GDP, consumption, investment and hours.) Table 3 reports the mean and median parameter estimates, and the standard deviation of the parameter estimates, across the sample of 50 estimates per model variant. The parameters are tightly estimated; mean and median parameter estimates are close to the true parameter values. ${ }^{17}$

\footnotetext{
${ }^{16}$ For the unpruned 'small shocks' sample paths, the KalmanQ filter and the QKF thus have similar performance; with $\mathrm{T}=100[\mathrm{~T}=500]$, the KalmanQ filter is more accurate (across all variables) than the QKF in about $45 \%$ [54\%] of the simulation runs.

17 A more detailed evaluation of the small sample properties of the QML estimator is left for future research.
} 
Table 3 RBC model: distribution of quasi-maximum likelihood estimates of model parameters based on the KalmanQ filter

\begin{tabular}{|c|c|c|c|c|c|c|}
\hline & $\sigma$ & $\eta$ & $\rho_{\theta}$ & $\rho_{\lambda}$ & $\sigma_{\theta}$ & $\sigma_{\lambda}$ \\
\hline & (1) & (2) & (3) & (4) & $(5)$ & (6) \\
\hline \multicolumn{7}{|c|}{ (a) Model variant with big shocks $\left(\sigma_{\theta}=0.20, \sigma_{\lambda}=0.01\right)$} \\
\hline True parameter value & 10.00 & 4.00 & 0.99 & 0.99 & $20.00 \%$ & $1.00 \%$ \\
\hline Mean estimate & 10.41 & 4.13 & 0.99 & 0.98 & $18.73 \%$ & $0.97 \%$ \\
\hline Median estimate & 10.09 & 4.01 & 0.99 & 0.98 & $18.71 \%$ & $0.92 \%$ \\
\hline Standard dev. of estimates & 1.36 & 1.53 & 0.003 & 0.005 & $1.44 \%$ & $0.21 \%$ \\
\hline \multicolumn{7}{|c|}{ (b) Model variant with small shocks $\left(\sigma_{\theta}=0.01, \sigma_{\lambda}=0.0005\right)$} \\
\hline True parameter value & 10.00 & 4.00 & 0.99 & 0.99 & $1.00 \%$ & $0.05 \%$ \\
\hline Mean estimate & 10.26 & 4.85 & 0.99 & 0.69 & $0.76 \%$ & $0.20 \%$ \\
\hline Median estimate & 10.15 & 3.40 & 0.99 & 0.69 & $0.74 \%$ & $0.18 \%$ \\
\hline Standard dev. of estimates & 1.20 & 3.39 & 0.005 & 0.26 & $0.12 \%$ & $0.14 \%$ \\
\hline
\end{tabular}

Note: The Table reports true parameter values, as well as the mean, median and standard deviation of quasi-maximum likelihood (QML) estimates of structural model parameters based on the KalmanQ filter (developed in this paper), obtained for 50 simulation runs with $\mathrm{T}=100$ periods of the 'big shocks' $\mathrm{RBC}$ model variant (Panel (a)) and for 50 simulation runs $(\mathrm{T}=100)$ of the 'small shocks' RBC model variant (Panel (b)). Four observables (GDP, consumption, investment and hours) are used for the QML estimation. $\sigma$ : risk aversion coefficient (Col. (1)); $\eta$ : Frisch labor supply elasticity (Col. (2)); $\rho_{\theta} \quad\left[\rho_{\lambda}\right]$ : autocorrelation of TFP [taste shock] (Cols. (3)-(4)); $\sigma_{\theta}\left[\sigma_{\lambda}\right]$ : standard deviation of TFP [taste shock] innovation (Cols. (5)-(6))

\subsection{State Equations with Randomly Drawn Coefficients}

Many other Monte Carlo experiments confirmed that the KalmanQ filter is competitive with the particle filter, in terms of accuracy of the estimated state variables. To document the performance of the filter in a broad range of setting, I applied it to simulated data generated using variants of the pruned state equations (3),(4) whose coefficients were drawn randomly from normal distributions. Tables 4 reports moments of the resulting simulated latent state variables, while Table 5 documents the accuracy of the filters. In both Tables, Panel (a) pertains to models with $n=20$ variables, while Panel (b) assumes $n=7$ variables; I refer to the models in Panels (a) and (b) as 'medium models', and as 'small models', respectively. In both set-ups, $m=7$ independent exogenous innovations are assumed, and the first four elements of the state vector $\omega_{t}$ are observed with measurement error $\left(n_{Y}=4\right)$. The standard deviations of the (independent) exogenous innovations $\left(\varepsilon_{t+1}\right)$ and of measurement errors $\left(\psi_{t}\right)$ are set at $1 \% .{ }^{18}$ The elements of $F_{0}$ are independent draws from $N(0,1)$ that are scaled by a common factor so that the largest element of $F_{0}$ is $(0.01)^{2}$ in absolute value. The elements of $F_{1}$ are independent draws from $N(0,1)$ that are scaled by a common factor so that the largest eigenvalue of $F_{1}$ has an absolute value of 0.99 . The elements of $F_{2}$ are independent draws from $N(0,1)$. In one set of simulations, referred to as 'strong cur-

\footnotetext{
$\overline{18} \Sigma_{\varepsilon}=(.01)^{2} I_{m} ; \Sigma_{\psi}=(.01)^{2} I_{n_{y}}$. As before, the parameter $\xi$ that scales the size of the shocks is normalized as $\xi=1$.
} 
Table 4 Models with randomly drawn coefficients: average standard deviations of state variables

\begin{tabular}{|c|c|c|}
\hline & Strong curvature & Weak curvature \\
\hline & (1) & (2) \\
\hline \multicolumn{3}{|l|}{ (a) Medium models $(\mathrm{n}=20)$} \\
\hline \multicolumn{3}{|l|}{ (a.1) Non-HP filtered variables } \\
\hline Second-order approximated model & 3.061 & 0.119 \\
\hline Linearized model & 0.106 & 0.106 \\
\hline \multicolumn{3}{|l|}{ (a.2) HP filtered variables } \\
\hline Second-order approximated model & 1.089 & 0.094 \\
\hline Linearized model & 0.092 & 0.092 \\
\hline \multicolumn{3}{|l|}{ (b) Small models $(n=7)$} \\
\hline \multicolumn{3}{|l|}{ (b.1) Non-HP filtered variables } \\
\hline Second-order approximated model & 2.446 & 0.147 \\
\hline Linearized model & 0.144 & 0.144 \\
\hline \multicolumn{3}{|l|}{ (b.2) HP filtered variables } \\
\hline Second-order approximated model & 0.678 & 0.116 \\
\hline Linearized model & 0.115 & 0.115 \\
\hline
\end{tabular}

Note: The Table reports average standard deviations of simulated state variables generated by pruned state equations (see (3), (4)) whose coefficients were drawn randomly from normal distributions. Panel (a) considers models with $n=20$ variables ('medium models'), while Panel (b) pertains to models with $n=7$ variables ('small models'). In Column (1), labeled 'Strong curvature', all elements of the matrices of curvature coefficients $F_{11}, F_{12}, F_{22}$ are independent draws from N(0,1); in Column (2), labeled 'Weak curvature', $F_{11}, F_{12}, F_{22}$ are independent draws from $N\left(0,(0.01)^{2}\right)$, so that curvature is much smaller, on average than in Column (1). (See Sect. 3.2 for further details.)

For both the 'medium' and 'small' model variants, 50 random 'strong curvature' coefficient sets, and 50 random 'weak curvature' coefficient sets were drawn. For each of the resulting 200 sets of coefficients, the model was simulated over $\mathrm{T}=100$ periods. For each simulation run, the standard deviation of each state variable (i.e. of each element of the vectors $\omega_{t}$ and $\omega_{t}^{(1)}$ ) was computed (without measurement error); then, standard deviations were averaged across all variables and coefficient draws, for each of the four model classes (medium/small models with strong/weak curvature), respectively. The Table shows those averaged standard deviations. (The averaged standard deviations are not reported in \%.)

The rows labeled 'second-order approximated model' and 'linearized model' show average standard deviations of elements of the state vectors $\omega_{t}$ and of $\omega_{t}^{(1)}$, respectively, where $\omega_{t}$ is generated using the pruned quadratic state equation (4), while $\omega_{t}^{(1)}$ is generated using the linearized state equation (3)

vature' simulations, all elements of $F_{11}, F_{12}, F_{22}$ are independent draws from $N(0,1)$; in another set of simulations with 'weak curvature', the elements of $F_{11}, F_{12}, F_{22}$ are independent draws from $N\left(0,(0.01)^{2}\right)$, so that curvature is much smaller, on average. For both the 'medium' and 'small' model variants, 50 random 'strong curvature' coefficient sets, and 50 random 'weak curvature' coefficient sets were drawn. Thus, 200 different random sets of coefficients $\left(F_{0}, F_{1}, F_{2}, F_{11}, F_{12}, F_{22}\right)$ are considered. For each set of coefficients, the state equations (3) and (4) were simulated over $\mathrm{T}=100$ periods (each run was initialized at the unconditional mean of the state vector), and the filters were applied to the observables (with measurement error).

Table 4 reports (averaged) standard deviations of the latent state variables for the 'medium' and 'small' model variants with 'strong curvature' (Col. (1)) and with 'weak 
Table 5 Models with randomly drawn coefficients: accuracy of filters

(a) Medium models $(n=20)$

\section{Average RMSES}

$\begin{array}{lll}\text { KalmanQ } & 0.155 & 0.0308 \\ \text { QKF } & - & 0.0320 \\ \text { PF (100,000) } & 31.243 & 0.0851 \\ \text { KalmanL } & 2.978 & 0.0523\end{array}$

\section{Maximum errors}

$\begin{array}{lll}\text { KalmanQ } & 4.784 & 0.2789 \\ \text { QKF } & - & 0.3612 \\ \text { PF }(100,000) & 4440.00 & 9.3808 \\ \text { KalmanL } & 149.441 & 1.2315\end{array}$

Fraction of runs in which RMSE is lower for KalmanQ than for other filters

$\begin{array}{lll}\text { QKF } & - & 0.38 \\ \text { PF }(100,000) & 1.00 & 0.98 \\ \text { KalmanL } & 1.00 & 0.94\end{array}$

Fraction of runs in which maximum error is lower for KalmanQ than for other filters

QKF

PF (100,000)

KalmanL

(b) Small models $(n=7)$

Average RMSES

$$
\text { KalmanQ }
$$

0.035

0.0184

QKF

PF (100,000)

0.204

0.0185

KalmanL

38.082

0.0186

1.651

0.0409

Maximum errors

KalmanQ
QKF

PF (100,000)

KalmanL

1.12

0.0943

41.93

0.1084

3263.28

0.0954

37.38

0.1265

Fraction of runs in which RMSE is lower for KalmanQ than for other filters
QKF
1.00
0.42
PF (100,000)
0.76
0.76
KalmanL
1.00
0.96

Fraction of runs in which maximum error is lower for KalmanQ than for other filters

\begin{tabular}{llr} 
QKF & 1.00 & 0.52 \\
PF $(100,000)$ & 0.82 & 0.50 \\
KalmanL & 1.00 & 0.66 \\
\hline
\end{tabular}


Table 5 continued

Note: The Table reports average Root Mean Squared Errors (RMSEs) and maximum estimation errors of estimated latent variables produced by four filters, across simulation runs and state variables, for versions of the pruned state equation (4) with randomly drawn coefficients; see Sect. 3.2. Panel (a) ('medium models') assumes $n=20$ variables; Panel (b) ('small model') assumes $n=7$ variables. In 'strong curvature' ['weak curvature'] experiments (see Column 1 [2]), all elements of the matrices of curvature coefficients $F_{11}, F_{12}, F_{22}$ are independent draws from $N(0,1)\left[N\left(0,(0.01)^{2}\right)\right]$. (See Sect. 3.2 for further details.) For both the 'medium' and 'small' model variants, 50 random 'strong curvature' coefficient sets, and 50 random 'weak curvature' coefficient sets were drawn. For each of the resulting 200 sets of coefficient, the model was simulated over $\mathrm{T}=100$ periods.

For each simulation run, the RMSE and the maximal error was computed, for each of the ' $n$ ' estimated latent variables; then, RMSEs were averaged across variables and coefficient draws, for each of the four model classes (medium/small models with strong/weak curvature); maximum errors were likewise determined across all $n$ variables, and across all draws, for each of the four model classes.

KalmanQ: the filter for pruned second-order models developed in this paper; $Q K F$ : Quadratic Kalman Filter (Ivashchenko 2014) that assumes that the unpruned second-order model (2) is the true DGP; $\operatorname{PF}(p)$ : standard particle filter with pparticles; KalmanL: standard Kalman filter that assumes that the linearized model is the true DGP.

The time paths for filtered estimates of the state variables generated by the QKF exploded in all 50 simulation runs of the 'medium models' with 'strong curvature'; thus, no RMSEs and Maximum Errors are reported for the Quadratic Kalman Filter (QKF) in Column (1) of Panel (a).

The QKF exploded in $48 \%$ of the 50 simulation runs of the 'small models' with 'strong curvature'; the RMSEs and Maximum Errors for the QKF reported in Column (1) of Panel (b) pertain to the $52 \%$ of the simulation runs in which the QKF did not explode; the reported fraction of runs in which RMSEs and Maximum Errors are lower for KalmanQ than for QKF likewise only pertains to the runs in which QKF did not explode.

The QKF did not explode in the simulation runs of the 'weak curvature' model variants (Column (2))

curvature' (Col. (2)). The rows labeled 'Second-order approximated model' and 'Linearized model' show (averaged) standard deviations of $\omega_{t}$ and of $\omega_{t}^{(1)}$, respectively, where $\omega_{t}$ was generated using the pruned quadratic state equation (4), while $\omega_{t}^{(1)}$ was generated using the linear state equation (3). ${ }^{19}$ In 'strong curvature' model variants, the average predicted volatility of $\omega_{t}$ (second-order accurate) is several times larger than that of $\omega_{t}^{(1)}$. By contrast, in the 'weak curvature' variants, the volatility of the second-order accurate variables is only slightly higher than that of the first-order accurate variables.

Table 5 compares the accuracy of the KalmanQ, QKF, PF(100,000) and KalmanL filters, for each of the four model classes (medium/small models with strong/weak curvature). For each model class, the KalmanQ filter generates lower average RMSEs and lower maximum errors than the PF $(100,000)$ and KalmanL filters. E.g., for the 'medium models', the average RMSEs of KalmanQ, PF(100,000) and KalmanL are $0.155,31.243$ and 2.978, respectively, under 'strong curvature', and 0.031, 0.085 and 0.052 , respectively under 'weak curvature' (see Panel (a)).

The time paths of filtered estimates of state variables generated by the QKF exploded in all simulation runs for 'medium models' with 'strong curvature'. For 'small models' with 'strong curvature', the QKF exploded in $48 \%$ of the simulation runs; for the

19 For each simulation run, the standard deviation of each element of $\omega_{t}$ and $\omega_{t}^{(1)}$ was computed; then, standard deviations were averaged across variables and coefficient draws, for each of the four model classes (medium/small models with strong/weak curvature). 
runs where the QKF did not explode, the KalmanQ filter is markedly more accurate than the QKF (see Column (1) of Panel (b), Table 5). In the 'weak curvature' model variants, by contrast, the QKF did not explode in any of the runs-KalmanQ is slightly more accurate than QKF in terms of average RMSEs and Maximum Errors across all simulation runs. ${ }^{20}$

KalmanQ, QKF, PF(100,000) and KalmanL require 0.10, 0.14, 24.78 and $0.02 \mathrm{~s}$, respectively, to filter simulated series of $\mathrm{T}=100$ periods generated by the 'small models'; for 'medium models' the corresponding computing times are $0.86,0.17,78.49$ and $0.03 \mathrm{~s}$, respectively. This confirms the finding that the KalmanQ filter is much faster than the particle filter. The KalmanQ filter is faster than the QKF for 'small models', but not for 'medium models'.

\section{Conclusion}

This paper has developed a novel approach for the estimation of latent state variables in DSGE models that are solved using a second-order accurate approximation and the 'pruning' scheme of Kim et al. (2008). By contrast to particle filters, no stochastic simulations are needed for the deterministic filter here; the present method is thus much faster than particle filters. The use of the pruning scheme distinguishes the filter developed here from Ivashchenko's (2014) deterministic Quadratic Kalman filter (QKF). The present filter performs well even in models with big shocks and high curvature. In Monte Carlo experiments, the filter developed here generates more accurate estimates of latent state variables than the standard particle filter, especially when the model has big shocks and high curvature. The present filter is also more accurate than a Kalman filter that treats the linearized model as the true DGP. Due to its high speed, the filter presented here is suited for the estimation of model parameters; a quasi-maximum likelihood procedure can be used for that purpose.

Acknowledgments I am very grateful to three anonymous referees for detailed and constructive comments. I also thank Martin Andreasen, Sergey Ivashchenko, Jinill Kim and Raf Wouters for useful discussions. Financial support from the National Bank of Belgium and from 'Action de recherche concertée' ARC-AUWB/2010-15/ULB-11 is gratefully acknowledged.

\section{Appendix: Computing Moments of the State Vector (for KalmanQ Filter Formula)}

The unconditional mean and variance of the state vector $Z_{t+1}$ of the augmented state equation (5) are given by: $E\left(Z_{t+1}\right)=\left(I-G_{1}\right)^{-1} G_{0}$ and $V\left(Z_{t+1}\right)=$ $G_{1} V\left(Z_{t+1}\right) G_{1}^{\prime}+V\left(u_{t+1}\right)$, respectively. Stationarity of $Z_{t+1}$ (which holds under the assumption that all eigenvalues of $F_{1}$ are strictly inside the unit circle) implies

\footnotetext{
${ }^{20} \mathrm{I}$ also examined filter performance using unpruned sample paths generated by the state equations with randomly drawn coefficients (results available in request). All 'strong curvature' model variants generated exploding unpruned sample paths. In the 'weak curvature' model variants, none of the unpruned sample paths exploded - pruned and unpruned sample paths were highly correlated; the QKF and KalmanQ filter showed similar performance.
} 
$E\left(Z_{t+1}\right)=E\left(Z_{t}\right), V\left(Z_{t+1}\right)=V\left(Z_{t}\right)$. Once $V\left(u_{t+1}\right)$ has been determined, $V\left(Z_{t+1}\right)$ can efficiently be computed using a doubling algorithm. Note that $\omega_{t}^{(1)}=$ $\sum_{i=0}^{\infty}\left(F_{1}\right)^{i} F_{2} \varepsilon_{t-i}$ and recall that

$$
u_{t+1} \equiv G_{2} \varepsilon_{t+1}+G_{12} \omega_{t}^{(1)} \otimes \varepsilon_{t+1}+G_{22}\left[P\left(\varepsilon_{t+1}\right)-E\left(P\left(\varepsilon_{t+1}\right)\right)\right] .
$$

$E\left(\omega_{t}^{(1)}\right)=0, E\left(\omega_{t}^{(1)} \otimes \varepsilon_{t+1}\right)=0, E\left(\left(\omega_{t}^{(1)} \otimes \varepsilon_{t+1}\right) \varepsilon_{t+1}^{\prime}\right)=0, E\left(\left(\omega_{t}^{(1)} \otimes \varepsilon_{t+1}\right) P\left(\varepsilon_{t+1}\right)^{\prime}\right)=0$ hold as $\varepsilon_{t+1}$ has mean zero and is serially independent. Hence, the covariances between the first and second right-hand side (rhs) terms in (A.1), and between the second and third rhs terms are zero. $\varepsilon_{t} \sim N\left(0, \Sigma_{\varepsilon}\right)$ implies that the unconditional mean of all third order products of elements of $\varepsilon_{t+1}$ is zero (Isserlis' theorem): $E \varepsilon_{t+1}^{i} \varepsilon_{t+1}^{j} \varepsilon_{t}^{k}=0$ for all $i, j, k=1, \ldots, \mathrm{m}$, where $\varepsilon_{t+1}^{h}$ is the $h$-th element of $\varepsilon_{t+1}$. Thus the covariance between the first and third rhs terms in (A.1) too is zero. Note that $V\left(\omega_{t}^{(1)} \otimes \varepsilon_{t+1}\right)=V\left(\omega_{t}^{(1)}\right) \otimes \Sigma_{\varepsilon}$, with $V\left(\omega_{t}^{(1)}\right)=F_{1} V\left(\omega_{t}^{(1)}\right) F_{1}^{\prime}+F_{2} \Sigma_{\varepsilon} F_{2}^{\prime}$. Thus,

$$
V\left(u_{t+1}\right)=G_{2} \Sigma_{\varepsilon} G_{2}^{\prime}+G_{12}\left(V\left(\omega_{t}^{(1)}\right) \otimes \Sigma_{\varepsilon}\right) G_{12}^{\prime}+G_{22} V\left(P\left(\varepsilon_{t+1}\right)\right) G_{22}^{\prime} .
$$

$\varepsilon_{t} \sim N\left(0, \Sigma_{\varepsilon}\right)$ also implies that the covariance between $\varepsilon_{t+1}^{i} \varepsilon_{t+1}^{j}$ and $\varepsilon_{t+1}^{r} \varepsilon_{t+1}^{s}$ is

$$
\operatorname{Cov}\left(\varepsilon_{t+1}^{i} \varepsilon_{t+1}^{j}, \varepsilon_{t+1}^{r} \varepsilon_{t+1}^{s}\right)=\sigma_{i, r} \sigma_{j, s}+\sigma_{i, s} \sigma_{j, r} \text { for i,j,r,s }=1, \ldots, \mathrm{m},
$$

where $\sigma_{i, r}=E\left(\varepsilon_{t+1}^{i} \varepsilon_{t+1}^{r}\right)$. (See, e.g., Triantafyllopoulos 2002). This formula allows to compute $V\left(P\left(\varepsilon_{t+1}\right)\right)$, the covariance matrix of the vector

$$
\begin{aligned}
P\left(\varepsilon_{t+1}\right) \equiv & \left(\varepsilon_{t+1}^{1} \varepsilon_{t+1}^{1}, \varepsilon_{t+1}^{1} \varepsilon_{t+1}^{2}, \ldots, \varepsilon_{t+1}^{1} \varepsilon_{t+1}^{m}, \varepsilon_{t+1}^{2} \varepsilon_{t+1}^{2}, \ldots, \varepsilon_{t+1}^{2} \varepsilon_{t+1}^{m}, \ldots,\right. \\
& \left.\varepsilon_{t+1}^{m-1} \varepsilon_{t+1}^{m-1}, \varepsilon_{t+1}^{m-1} \varepsilon_{t+1}^{m}, \varepsilon_{t+1}^{m} \varepsilon_{t+1}^{m}\right) .
\end{aligned}
$$

\section{Conditional Variance of State-Space Disturbance}

To derive the formula for the conditional variance of $u_{t+1}[(10)$ in main text $]$ these facts are used:

(i) $E\left(\left(\omega_{t}^{(1)} \otimes \varepsilon_{t+1}\right) \varepsilon_{t+1}^{\prime} \mid \Upsilon^{t}\right)=\omega_{t, t}^{(1)} \otimes \Sigma_{\varepsilon}$, with $\omega_{t, t}^{(1)} \equiv E\left(\omega_{t}^{(1)} \mid \Upsilon^{t}\right)$.

(ii) $E\left(\left(\omega_{t}^{(1)} \otimes \varepsilon_{t+1}\right)\left(\omega_{t}^{(1)} \otimes \varepsilon_{t+1}\right)^{\prime} \mid \Upsilon^{t}\right)=E\left(\left(\omega_{t}^{(1)} \omega_{t}^{(1) \prime}\right) \otimes\left(\varepsilon_{t+1} \varepsilon_{t+1}^{\prime}\right) \mid \Upsilon^{t}\right)=$ $E\left(\left(\omega_{t}^{(1)} \omega_{t}^{(1) \prime}\right) \mid \Upsilon^{t}\right) \otimes \Sigma_{\varepsilon}=\left(V_{t, t}^{\omega^{(1)}}+\omega_{t, t}^{(1)} \omega_{t, t}^{(1)} \prime\right) \otimes \Sigma_{\varepsilon}$. (Note that $\left.V_{t, t}^{\omega^{(1)}}=E\left(\omega_{t}^{(1)} \omega_{t}^{(1) \prime} \mid \Upsilon^{t}\right)-E\left(\omega_{t}^{(1)} \mid \Upsilon^{t}\right) E\left(\omega_{t}^{(1)} \mid \Upsilon^{t}\right)^{\prime}=E\left(\omega_{t}^{(1)} \omega_{t}^{(1) \prime} \mid \Upsilon^{t}\right)-\omega_{t, t}^{(1)} \omega_{t, t}^{(1) \prime}.\right)$

(iii) $E\left(P\left(\varepsilon_{t+1}\right) \varepsilon_{t+1}^{\prime} \mid \Upsilon^{t}\right)=0, \quad E\left(P\left(\varepsilon_{t+1}\right)\left(\omega_{t}^{(1)} \otimes \varepsilon_{t+1}\right)^{\prime} \mid \Upsilon^{t}\right)=0$ (due to Isserlis' theorem). Thus, the conditional covariance between the $1^{\text {st }}$ and $3^{\text {rd }}$ rhs terms in (A.1) and between the 2nd and 3rd rhs terms is zero. 


\section{References}

An, S., \& Schorfheide, F. (2007). Bayesian analysis of DSGE models. Econometric Reviews, 26, 113-172. Andreasen, M. (2012). Non-linear DSGE models and the central difference Kalman filter. Journal of Applied Econometrics, 28, 929-955.

Andreasen, M., Fernández-Villaverde, J., \& Rubio-Ramírez, J. (2013). The pruned state-space system for non-linear DSGE models: Theory and empirical applications. NBER Working Paper No. 18983.

Aruoba, S., Bocola, L., \& Schorfheide, F. (2012). A new class of nonlinear time series models for the evaluation of DSGE models. Working Paper, University of Maryland and University of Pennsylvania.

Collard, F., \& Juillard, M. (2001). Perturbation methods for rational expectations models. Working Paper, GREMAQ and CEPREMAP.

Del Negro, M., \& Schorfheide, F. (2011). Bayesian macroeconometrics. In J. Geweke, G. Koop, \& H. van Dijk (Eds.), The Oxford handbook of Bayesian econometrics (pp. 293-389). Oxford: Oxford University Press.

Fernández-Villaverde, J., \& Rubio-Ramírez, J. (2007). Estimating macroeconomic models: A likelihood approach. Review of Economic Studies, 74, 1059-1087.

Hamilton, J. (1994). Time series analysis. Princeton: Princeton University Press.

Harvey, A. (1989). Forecasting-structural time series models and the Kalman filter. Cambridge: Cambridge University.

Ivashchenko, S. (2014). DSGE model estimation on the basis of second-order approximation. Computational Economics, 43, 71-82

Jin, H., \& Judd, K. (2000). Applying PertSolv to complete market RBC models. Working paper, Stanford University.

Julier, S., \& Uhlmann, J. (2004). Unscented filtering and nonlinear estimation. Proceedings of the IEEE, 92, 401-422.

Kim, J., Kim, S., Schaumburg, E. \& Sims, C. (2008). Calculating and using second-order accurate solutions of discrete-time dynamic equilibrium models. Journal of Economic Dynamics and Control, 32, 33973414.

Kollmann, R. (1996). Incomplete asset markets and the cross-country consumption correlation puzzle. Journal of Economic Dynamics and Control, 20, 945-962.

Kollmann, R. (2002). Monetary policy rules in the open economy: Effects of welfare and business cycles. Journal of Monetary Economics, 49, 989-1015.

Kollmann, R. (2004). Solving non-linear rational expectations models: Approximations based on Taylor expansions. Working Paper, University of Paris XII.

Kollmann, R., Kim, S., \& Kim, J. (2011). Solving the multi-country real business cycle model using a perturbation method. Journal of Economic Dynamics and Control, 35, 203-206.

Lombardo, G., \& Sutherland, A. (2007). Computing second-order accurate solutions for rational expectation models using linear solution methods. Journal of Economic Dynamics and Control, 31, 515-530.

Norgaard, M., Poulsen, N., \& Ravn, O. (2000). New developments in state estimation for nonlinear systems. Automatica, 36, 1627-1638.

Schmitt-Grohé, S., \& Uribe, M. (2004). Solving dynamic general equilibrium models using a second-order approximation to the policy function. Journal of Economic Dynamics and Control, 28, 755-775.

Sims, C. (2000). Second order accurate solution of discrete time dynamic equilibrium models. Working Paper, Economics Department, Princeton University.

Smets, F., \& Wouters, R. (2007). Shocks and Frictions in US business cycles: A Bayesian DSGE approach. American Economic Review, 97, 586-660.

Triantafyllopoulos, K. (2002). Moments and cumulants of the multivariate real and complex Gaussian distributions. Working Paper, University of Bristol. 